PHYSICAL REVIEW FLUIDS 1, 050511 (2016)

\title{
Turning on a dime: Asymmetric vortex formation in hummingbird maneuvering flight
}

\author{
Yan Ren, ${ }^{1}$ Haibo Dong, ${ }^{1}$ Xinyan Deng, ${ }^{2}$ and Bret Tobalske ${ }^{3}$ \\ ${ }^{1}$ Department of Mechanical \& Aerospace Engineering, University of Virginia, Charlottesville, \\ Virginia 22903, USA \\ ${ }^{2}$ School of Mechanical Engineering, Purdue University, West Lafayette, Indiana 47907, USA \\ ${ }^{3}$ College of Humanities and Sciences, University of Montana, Missoula, Montana 59812, USA \\ (Received 12 August 2016; published 12 September 2016)
}

This paper is associated with a video winner of a 2015 APS/DFD Gallery of Fluid Motion Award. The original video is available from the Gallery of Fluid Motion, http://dx.doi.org/10.1103/APS.DFD.2015.GFM.V0088

DOI: 10.1103/PhysRevFluids.1.050511

A pure yaw turn of a calliope hummingbird is investigated through high-speed photogrammetry, three-dimensional surface reconstruction, and computational fluid dynamics simulations. We took high-speed videos of freely flying hummingbirds using three synchronized Photron high-speed cameras. The hummingbird body and wing motions were then reconstructed using a joint-based hierarchical subdivision surface method [1,2]. As an example, Fig. 1(a) shows a high-speed image of the maneuvering hummingbird and Fig. 1(b) presents the corresponding reconstructed model. There are in total six strokes of this flight and they can be divided into three flying phases: a starting phase (first stroke), a maneuvering phase (second to fourth strokes), and a recovering phase (fifth and sixth strokes).

A Cartesian grid based immersed boundary Navier-Stokes solver is used to simulate the unsteady flows around the body and wings of the bird $[3,4]$. We first look at the near wake structures by cutting two-dimensional flow slices along the hummingbird wing span. As shown in Fig. 1(c), leading edge vortex (LEV) structures at five flow slices can be observed for each wing. The contours of the LEV represent the normalized spanwise vorticity, which reveals the LEV strength. From the contour we observe that the LEV strength of the inner wing is greater than that of the outer wing. We further quantify the LEV circulation in the maneuvering phase. The results show that the inner wing produces $21 \%$ and $26 \%$ more average LEV circulation than the outer wing does during the downstrokes and upstrokes, respectively.

The three-dimensional wake structures are identified by plotting the isosurface of $Q$ criterion of the flow. As shown in Fig. 1(d), asymmetric wake structures between the inner and outer sides of the hummingbird body can be identified. Two new vortices, a shed trailing edge vortex (STEV) and a shed leading edge vortex (SLEV), can be identified at the outer side of the hummingbird body below the LEV. The LEV, tip vortex (TV), and STEV are connected to form a vortex ring near the outer wing tip region. Also, the LEV, SLEV, trailing edge vortex (TEV), and root vortex (RV) are connected to form another vortex ring. The two vortex rings form a unique dual-ring vortex structure at the outer side of the hummingbird body. In contrast, the LEV, TV, TEV, and RV are connected to form a single-ring vortex structure at the inner side of the hummingbird body.

In order to better understand the wake topology and its effect on the aerodynamic performance, nondimensional pressure is plotted to indicate the low-pressure regions behind the wings of the

Published by the American Physical Society under the terms of the Creative Commons Attribution 3.0 License. Further distribution of this work must maintain attribution to the author(s) and the published article's title, journal citation, and DOI. 


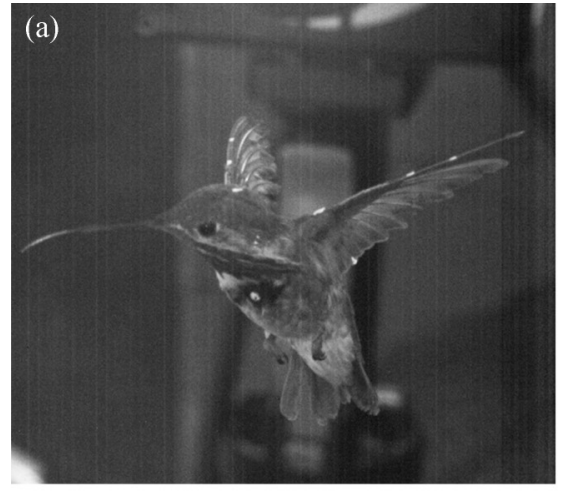

(c)

(d)

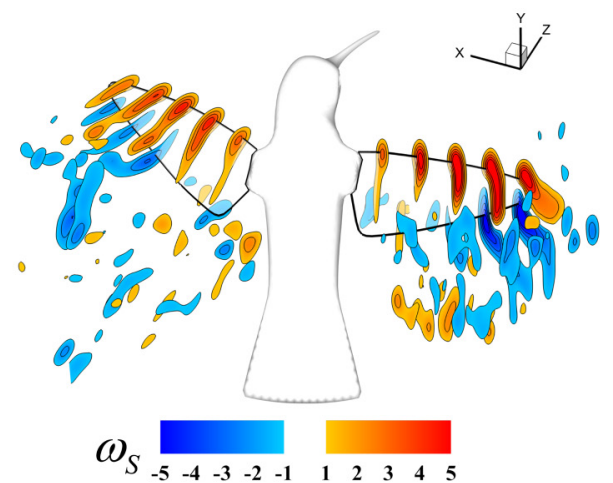

(b)
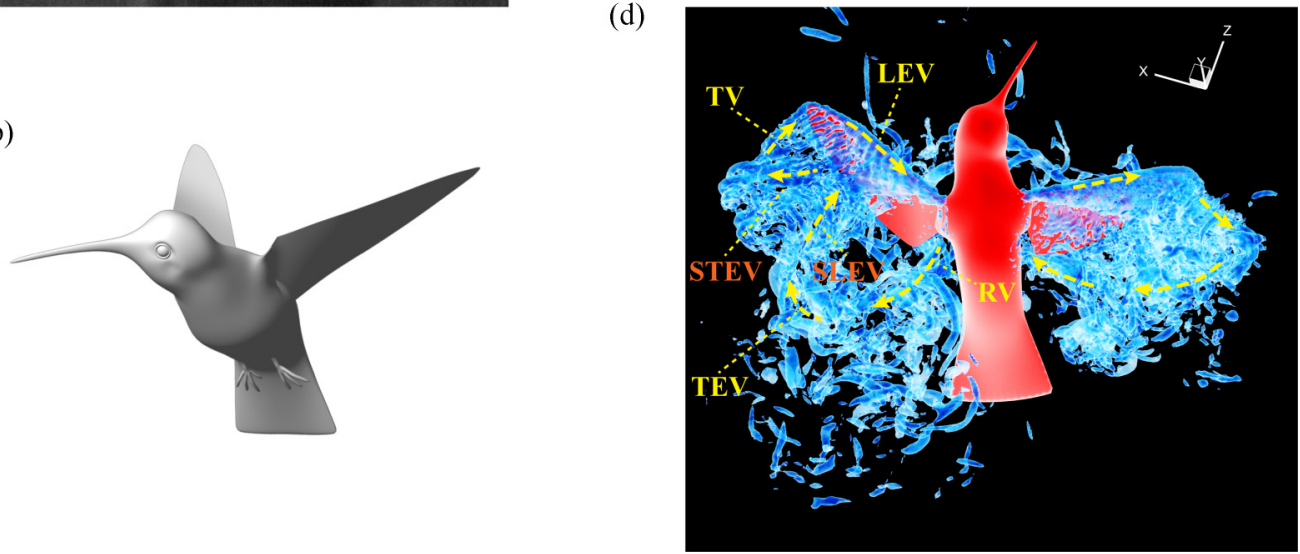

FIG. 1. Comparison of (a) a high-speed image of a real maneuvering hummingbird and (b) the corresponding reconstructed model. (c) Near wake structures and (d) 3D vortex structures at a typical time instance chosen in the maneuvering phase (the third stroke $t / T=0.33$ ). DOI: http://dx.doi.org/10.1103/APS.DFD.2015.GFM.V0088

hummingbird. Plotted in Fig. 2(a) is one isosurface of the pressure corresponding to a nondimensional pressure value of -1.0 . Noticeable in the figure is a large region of low pressure right behind the wings. However, for the outer side, the pressure isosurface shows branches near the midwing region,
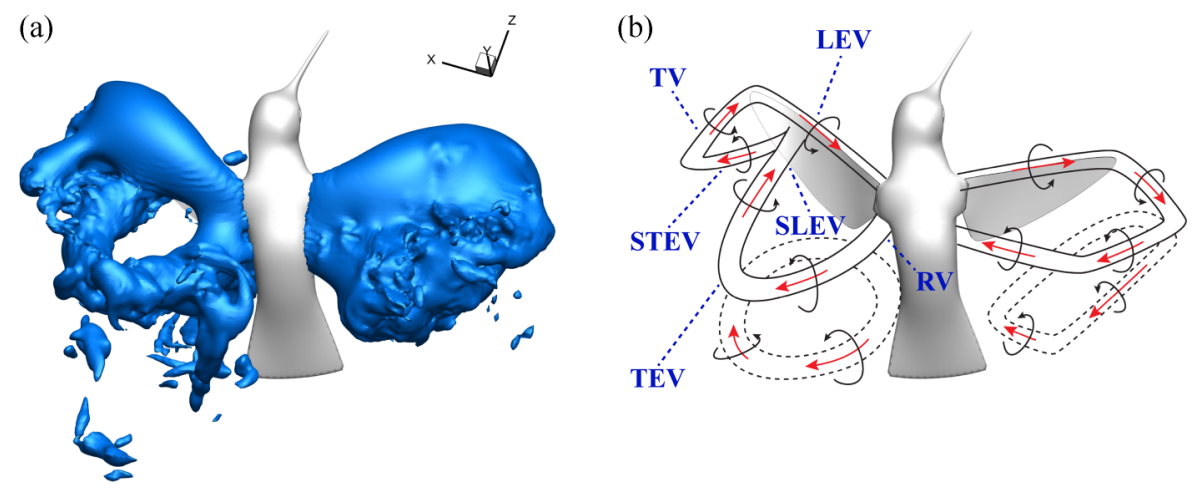

FIG. 2. (a) Isosurface of pressure corresponding to a nondimensional value of -1.0 and (b) the corresponding wake topology schematic at a typical time instance chosen in the maneuvering phase (the third stroke $t / T=0.33)$. 


\section{TURNING ON A DIME: ASYMMETRIC VORTEX ...}

which are due to the dual-ring vortex structure. At the other side, no branches of pressure isosurface can be observed behind the wings. The figure shows strong asymmetry of the pressure behavior between the inner and outer sides of the hummingbird body. Figure 2(b) shows the schematic of the correlation between identifiable vortex structures and regions of low pressure shown in Fig. 2(a). The LEV, STEV, SLEV, TV, TEV, and RV can be clearly identified in Fig. 2(b). The topology and formation of the dual-ring vortex structure are clearly revealed. More importantly, the dual-ring vortex structure corresponds to lower drag production compared to the single-ring vortex structure (31\% lower in this case). The drag difference between the two wings creates torque to sustain the yaw turn of the maneuvering hummingbird.

This work was supported by NSF Grant No. CBET-1313217 and AFOSR Grant No. FA9550-12$1-007$.

[1] C. Koehler, Z. Liang, Z. Gaston, H. Wan, and H. Dong, 3D reconstruction and analysis of wing deformation in free-flying dragonflies, J. Exp. Biol. 215, 3018 (2012).

[2] S. M. Walker, A. L. R. Thomas, and G. K. Taylor, Photogrammetric reconstruction of high-resolution surface topographies and deformable wing kinematics of tethered locusts and free-flying hoverflies, J. R. Soc. Interface 6, 351 (2009).

[3] Y. Ren and H. Dong, Proceedings of the 30th AIAA Applied Aerodynamics Conference (AIAA, Reston, 2012).

[4] Y. Ren, G. Liu, and H. Dong, Proceedings of the 53rd AIAA Aerospace Sciences Meeting (AIAA, Reston, 2015). 